\title{
An evaluation of the posterior cruciate ligament function in total knee arthroplasty with regard to its morphology and clinical properties
}

\author{
D. Marczak ${ }^{1}$, J. Kowalczewski ${ }^{1}$, T. Okoń1 ${ }^{1}$ M. Synder ${ }^{2}$, M. Sibiński² \\ ${ }^{1}$ Postgraduated Medical Education Centre, Orthopaedic Department, Otwock, Poland \\ ${ }^{2}$ Department of Orthopaedics and Paediatric Orthopaedics, Medical University of Lodz, Poland
}

[Received: 31 May 2016; Accepted: 1 August 2016]

\begin{abstract}
The aim of the study was to determine the degree of posterior cruciate ligament $(P C L)$ degeneration and the reduction in the number of its mechanoreceptors, in patients with advanced degenerative joint disease. PCLs taken from study group of 50 patients in the mean age of 70.7 (53-84) years with a diagnosis of advanced idiopathic osteoarthritis undergoing condylar total knee arthroplasty were compared to those taken form the control group of 10 knee joints of cadavers. Groups were matched with regard to sex and age. Histological examination of PCLs of the study group showed changes of an inflammatory process and no significant signs of osteoarthritis in the control group. A close correlation was found between the severity of degenerative changes on the $X$-ray images according to the Ahlbäck scale, and the increased mucoid degeneration $(p<0.0001)$, the severity of the degeneration of the collagen structure $(p<0.0001)$ and the presence of proprioceptors of PCLS $(p<0.0001)$. Conserving the PCL by the use of type cruciate retaining knee arthroplasty does not guarantee the preservation of correct proprioceptive sensation. (Folia Morphol 2017; 76, 1: 94-99)
\end{abstract}

Key words: posterior cruciate ligament, osteoarthritis, knee replacement, proprioception, function

\section{INTRODUCTION}

The literature presents no clear position regarding the suitability and differences between two basic types of total knee arthroplasty (TKA): cruciate retaining (CR) and the posteriori stabilised (PS). It is very difficult to distinguish the survival length and kinematics of implants manufactured by various firms with regard to the material used, the shape of the polyethylene insert and the shape of the femoral element. The continual development and improvement of both types of implants, together with the introduction of such surgical techniques as computer navigation and "patient-matched instrumentation", has led to a lack of fully objective studies comparing the two types of implants, and hence, an absence of strict guidelines for applying types of TKA in the treatment of osteoarthritis. The main difference between the two types of condylar TKA is based on the presence or absence of the PCL. It may be left intact, as is the case with the CR TKA, or replaced by a tibial insert and shape of femoral element, as in the PS TKA. Therefore, the key to the matter is the role of the PCL in TKA.

The thesis of the paper assumes that if osteoarthritis affects all the elements of the knee joint, it

Address for correspondence: M. Sibiński, MD, PhD, Department of Orthopaedics and Paediatric Orthopaedics, Medical University of Lodz, ul. Pomorska 251, 92-213 kódź, Poland, tel: +48 42 2014285, e-mail: sibinskimar@gmail.com; marcin.sibinski@umed.lodz.pl 
should also include the posterior cruciate ligament $(\mathrm{PCL})$, and should influence its proprioceptive and biomechanical properties. For this reason, the PCL will not provide sufficient stability for the knee after TKA, particularly in patients with advanced arthrosis. In this case, it is more justifiable to use the PS type TKA, which thanks to its construction, replaces the biomechanical function of the PCL.

The aim of the study was to determine the degree of PCL degeneration, and the reduction in the number of its mechanoreceptors, according to microscope examination in patients undergoing TKA for advanced degenerative joint disease. The study also compares these results with those of cadaver knees without osteoarthritis.

\section{MATERIALS AND METHODS}

The study group comprised 50 patients (40 women and 10 men) with a diagnosis of idiopathic osteoarthritis undergoing PS-type condylar TKA. The age of the patients ranged from 53 to 84 (mean 70.7) years. Each patient was subjected to $X$-ray examination of the knee joint in two projections: antero-posterior (AP) and lateral in a standing position.

The control group comprised 10 PCLs taken from 10 knee joints of cadavers. The cadavers were matched to those of the study group with regard to sex and age ( 7 women and 3 men, aged from 67 to 78 [mean 72] years). No degenerative changes were noted in the knee joints in the control group. No statistically significant differences were noted between the study group and the control group with regard to age $(p=0.94)$ or gender $(p=0.78)$.

The following inclusion criteria were applied: informed consent of the patient to participate in the study, no evidence of posterior instability of the knee during clinical examination (posterior drawer test), no evidence of macroscopic damage to the PCL during surgery or autopsy, radiological documentation of advanced idiopathic osteoarthritis of the knee in the study group. The study excluded patients with rheumatoid arthritis (RA) and other systemic connective tissue diseases, post-traumatic osteoarthritis, as well as vascular and metabolic diseases likely to have an impact on the quality of the PCL.

Degenerative changes to the joints were rated according to the Ahlbäck radiological scale [1]. Twenty patients were found to have grade 3 changes, 19 with grade 4 and 11 with grade 5 .

The PCL preparations were taken and prepared for histological examination, identical procedures being used for both the study and control groups. All were subjected to haematoxylin and eosin, Alcian blue, Gomori and van Gieson staining, and an immunohistochemical method using anti-S-100 antibodies [8].

Mucoid degeneration of the ligament was evaluated by Alcian blue dye. The following scale was used for grading the degree of degenerative change: grade 0 - fully functioning ligament; grade 1 - a single mucoid change; grade 2 - some mucoid degeneration; grade 3 - clear mucoid degeneration; grade 4 - massive mucoid degeneration with the presence of small individual mucoid lakes; grade 5 - massive mucoid degeneration with the presence of large number of mucoid "lakes".

Van Gieson and Gomori staining was used to determine the severity of collagen degeneration according to the following scale: grade 0 - regular ligament; grade $1-$ a small undulation of the collagen fibres, without fibration or disintegration; grade 2 - a small undulation of collagen fibres with fibration and disintegration; grade 3 - a clear undulation of collagen fibres with fibration and disintegration; grade 4 - significant undulation with stratification and disintegration.

Immunohistochemical staining with DAKO S-100 antibodies. The following scale was used to evaluate the number of neurofilaments: grade 0 - no neurofilaments; grade 1 - a few neurofilaments; grade 2 - a large number of neurofilaments.

\section{Statistical analysis}

Statistica 9.0 (StatSoft) was used for all statistical analyses. The Mann-Whitney test was used to test the difference in age between patients and controls, as well as the differences of PCLs between patients and controls. The $\chi^{2}$ test with Yeats correction was used to evaluate differences in gender distribution between the study and control groups. The Spearman's rank test was used to examine the correlation between the severity of degenerative changes and the severity of the mucoidal degeneration of the PCL (Alcian blue stain), and the severity of the degeneration of the collagen structure of the PCL (Gomori and van Gieson stain). The level of significance was regarded as $p<0.05$.

\section{RESULTS}

Routine staining with haematoxylin and eosin was used to reveal the histological appearance of the ligament. The presence of blood vessel and mononuclear 
Table 1. A comparison of the degree of mucoid degeneration, degree of collagen degeneration and the presence of neurofilaments with Ahlbäck score, indicating the number of preparations in the posterior cruciate ligament test group and control group

\begin{tabular}{lccccccc}
\hline & Scale & $\begin{array}{c}\text { Ahlbäck } \\
\mathbf{3}\end{array}$ & $\begin{array}{c}\text { Ahlbäck } \\
\mathbf{4}\end{array}$ & $\begin{array}{c}\text { Ahlbäck } \\
\mathbf{5}\end{array}$ & $\begin{array}{c}\text { Ahlbäck } \\
\text { vs. parameters } \\
\text { in the study group }\end{array}$ & $\begin{array}{c}\text { Control } \\
\text { group }\end{array}$ & $\begin{array}{c}\text { Control } \\
\text { vs. study } \\
\text { group }\end{array}$ \\
\hline $\begin{array}{l}\text { Degree of mucoid } \\
\text { degeneration }\end{array}$ & $0^{\circ}$ & 0 & 0 & 0 & & 2 & \\
& $1^{\circ}$ & 5 & 1 & 0 & & 8 & \\
& $2^{\circ}$ & 9 & 4 & 1 & $\mathrm{p}<0.0001$ & 0 & $\mathrm{p}<0.0001$ \\
& $3^{\circ}$ & 5 & 7 & 2 & & 0 & \\
& $4^{\circ}$ & 1 & 6 & 8 & & 0 & \\
& $5^{\circ}$ & 0 & 1 & 0 & & 0 & \\
\hline Degree of collagen & $0^{\circ}$ & 0 & 0 & 0 & & 8 & \\
degeneration & $1^{\circ}$ & 2 & 0 & 0 & & 2 & \\
collagen FlBRES & $2^{\circ}$ & 13 & 3 & 0 & $\mathrm{p}<0.0001$ & 0 & $\mathrm{p}<0.0001$ \\
& $3^{\circ}$ & 5 & 10 & 2 & & 0 & \\
& $4^{\circ}$ & 0 & 6 & 9 & & 0 & \\
\hline Presence of & $0^{\circ}$ & 0 & 16 & 11 & & 0 & \\
neurofilaments & $1^{\circ}$ & 13 & 3 & 0 & $\mathrm{p}<0.0001$ & 3 & $\mathrm{p}<0.0001$ \\
& $2^{\circ}$ & 7 & 0 & 0 & & 7 & \\
\hline
\end{tabular}

cell proliferation, cartilage or bone metaplasia was revealed. In all samples, the study group showed changes of an inflammatory process; however, no significant signs of osteoarthritis were found in any of the PCLs from the control group. As there was no possibility to systematically assess the degree of change, the results of this test were not intended for statistical analysis. The results of the Alcian blue and van Gieson and Gomori staining, as well as the S-100 antibody analysis, are shown in Table 1.

A close correlation was found between the severity of degenerative changes identified in the X-ray images according to the Ahlbäck scale, and the severity of the mucoid degeneration of the $P C L$ identified by Alcian blue staining ( $p<0.0001$, test value 0.615 ), the severity of the degeneration of the collagen structure of the PCL (Gomori and van Gieson stains) $(p<0.0001$, test value 0.759 ) and the presence of neural elements in the ligament samples (anti-S-100) $(p<0.0001$, test value 0.844 ). Samples graded higher according to the Ahlbäck scale possessed fewer receptors, as revealed by staining of antibodies.

A statistically significant difference was also found between the study and control groups with regard to the degree of mucoid degeneration, degree of collagen degeneration and occurrence of proprio- ceptors/neural elements $(p<0.0001, p<0.0001$, $p<0.0001$, respectively).

\section{DISCUSSION}

Posterior cruciate ligament samples taken from patients with degenerative disease of the knee undergoing TKA were evaluated by microscope. In these tests, it was found that the mucoid degeneration of the ligament was related to the stratification and destruction of collagen fibres. Similar changes have been described previously by Nelissen and Hogendorn [13] in 20 PCL samples taken during TKA. They report mucoid degeneration and abnormal course, with disturbances in the spatial structure of the collagen fibres. However, the interpretation of these results is complicated by the lack of homogeneity of the study group, of which almost half (9 of 20) is composed of patients with RA. In addition, it is practically impossible to extrapolate any meaningful results from the control group, which consisted of 1 person and was unrepresentative of the age of the study group. A similar study by Klainbart et al. [11] compares the histological images of 24 PCLs taken from 24 patients during TKA of the knee with those of $36 \mathrm{PCL}$ taken from cadavers or amputated limbs, with the study and control groups being matched for age. As noted 
in the present work, Klainbart et al. [11] revealed significantly greater degenerative changes to the $\mathrm{PCL}$ in the study group compared with the control group. This suggests a reduction in the mechanical properties of the PCL in osteoarthritis patients compared to those in a population of a similar age.

An attempt to compare the macroscopic and histological pictures of both cruciate ligaments in osteoarthritic knee joints was made by Allain et al. [3]. Of 52 PCLs evaluated macroscopically as healthy, only 22 did not demonstrate changes under histological examination, which gives a clear indication of the difficulty of deciding whether to preserve the PCL during TKA based only on the evidence of macroscopic changes. This lack of correlation between the degree of histological degeneration in the PCL during osteoarthritis and macroscopic assessment has also been confirmed by Akisue et al. [2] and by Stubbs et al. [21]. After evaluating the histological changes to 50 PCLs, the latter report that PCLs typically exhibit varying degrees of degenerative histological changes. However, the degree of these changes observed under microscopic examination is not a suitable basis for making an intraoperative assessment of patients with degenerative disease. This represents a significant problem for the surgeon in pre- and intraoperative planning [21].

Radiological imaging is crucial in preoperative planning. More advanced changes identified by radiography are associated with greater mucoid degeneration of the ligaments and greater stratification and serious destruction of collagen fibres. The presence of a significant correlation between histological changes occurring in the PCL and the degree of changes observed radiologically would be a valuable one: it would allow the surgeon to estimate the degree of $\mathrm{PCL}$ degeneration on the basis of pre-operative $\mathrm{X}$-ray images and to decide on the possible removal of the degenerate PCL and make an appropriate choice of prosthesis. However, no such correlation was demonstrated by Stubbs et al. [21] in an evaluation of the degree of advanced degenerative disease.

Proponents of conserving the PCL during arthroplasty cite its role in proprioception of the knee joint. The first study on the presence of mechanoreceptors in the ligaments of the knee joint was published by Schultz et al. [15]. They show nerve endings to be present in anterior cruciate ligament and PCL preparations, while also observing a reduction in the number of mechanoreceptors in degenerative diseases of the knee. The presence of proprioceptors in the PCL has been demonstrated by a number of authors using various staining methods $[9,13]$. Barrett et al. [4] and Skinner et al. [19] describe a weakening of proprioception to be associated with age and with greater degenerative disease.

Simmons et al. [18] examined the role of proprioception in knee replacement in a group of 28 patients aged between 50 and 80 years of age with a very good post-operative clinical score who underwent the procedure. In 15 patients, the PCL was not removed and a CR endoprosthesis was implanted, while in the other 13, the PCL was removed and a PS implant inserted. All patients received very good results on the Knee Society Score (KSS) scale. Patients with grade II degenerative changes were mainly those who had received a CR endoprosthesis, and patients with stage III PS. Proprioception was rated on a special device in all patients (proprioception testing device), with no significant difference being identified between the patient groups. However, among patients with a significant degree of degenerative changes (grade III), those with a PS endoprosthesis demonstrated significantly better proprioception than those who had received a CR implant. For patients with grade II degeneration, no statistically significant differences in proprioception were found between those with PS and those with CR implants [18].

Similar results were obtained by Lattanzio et al. [12], who compared the proprioceptive function of 20 patients undergoing TKA: some by implantation of $\mathrm{PS}$, and some by CR. Conserving the PCL was found to have no positive effect on the proprioceptive function of the artificial joint. Cash et al. [6] also compared proprioception, and noted no difference in the feeling of knee flexion in two 30-person groups after a minimum of a year following implantation of either PS or CR endoprostheses. Swanik et al. [22] rated proprioception in 20 patients after total CR and PS arthroplasty. A small improvement in proprioception, as well as range of motion and stability of the artificial joint, was noted in all patients. No significant difference was noted between patients in whom the PCL had not been removed and in those after PS implantation.

The weakening of the structure of the PCL and its proprioceptive function in osteoarthritis has a significant impact on postoperative outcome of the artificial joint. Preserving a deficient PCL eventually leads to the loss of stability of the artificial joint and rapid destruc- 
tion of the polyethylene insert [16]. In addition, other arguments have been proposed against leaving the PCL intact during TKA. During surgery, unintended injury to the $P C L$ often occurs by resection of the proximal tibia [17]. Additional PCL injuries can occur during an attempt to equalise flexion and flexion after resection of the articular ends of the bones forming the knee joint, which is a difficult technique [20]. If the $\mathrm{PCL}$ is too tensed, the flexion space is less than the extension space. This leads to a restriction in the range of flexion and increases the stress shielding, affecting the operation of the posterior part of the tibial tray, in turn leading to faster aseptic loosening [16]. In correctly balancing the PCL, the surgeon is often forced to partially loosen the PCL with the aim of achieving equal flexion and extension spaces.

A number of authors who have performed studies on balancing the kinematics of PS and CR endoprostheses have shown that removal of the PCL and the implantation of a PS endoprosthesis not only increases the range of motion, but also prevents dislocation and makes walking upstairs more comfortable [14, 24]. Some works have been published which reveal no significant difference in clinical outcome between CR and PS TKA $[5,7,10,23]$. It can be concluded that the removal of the PCL does not result in deterioration of proprioception, range of motion or patient satisfaction. Arthroplasty with removal of the PCL is technically easier than the use of CR and gives longterm results which are equal, or often better, in terms of movement [14, 24].

The relationship between histological changes to changes observed in preoperative radiographic examination is of great importance in obtaining good long-term postoperative results. This work confirms the existence of a significant correlation between the histological changes taking place in the PCL and the level of radiological changes graded according to the Ahlbäck scale. These findings will enable the surgeon to predict the destruction of the PCL on the basis of pre-operative $\mathrm{X}$-ray images. They will also allow a pre-operative decision to be made regarding removal of the degenerate $P C L$ and implantation of a PS-type endoprosthesis.

\section{CONCLUSIONS}

A close correlation exists between the level of advanced degenerate changes in the knee joint according to radiological examination, and increased mucoid degeneration, increased degeneration of the collagen structure, and reduction in the number of proprioceptive nerve elements in the examined PCL. Conserving the PCL by the use of type CR knee arthroplasty does not guarantee the preservation of correct proprioceptive sensation.

\section{REFERENCES}

1. Ahlbäck S. Osteoarthrosis of the knee. A radiographic investigation. Acta Radiol Diagn (Stockh), 1968; Suppl 277: 7-72.

2. Akisue T, Stulberg BN, Bauer TW, et al. Histologic evaluation of posterior cruciate ligaments from osteoarthritic knees. Clin Orthop Relat Res. 2002(400): 165-173, doi: 10.1097/00003086-200207000-00021, indexed in Pubmed: 12072759.

3. Allain J, Goutallier D, Voisin MC. Macroscopic and histological assessments of the cruciate ligaments in arthrosis of the knee. Acta Orthop Scand. 2001; 72(3): 266-269, doi: 10.1080/00016470152846592, indexed in Pubmed: 11480602.

4. Barrett DS, Cobb AG, Bentley G. Joint proprioception in normal, osteoarthritic and replaced knees. J Bone Joint Surg Br. 1991; 73(1): 53-56, doi:10.4172/21577595.1000119, indexed in Pubmed: 1991775.

5. Bolanos AA, Colizza WA, McCann PD, et al. A comparison of isokinetic strength testing and gait analysis in patients with posterior cruciate-retaining and substituting knee arthroplasties. J Arthroplasty. 1998; 13(8): 906-915, doi: 10.1016/s0883-5403(98)90198-x, indexed in Pubmed: 9880184.

6. Cash RM, Gonzalez MH, Garst J, et al. Proprioception after arthroplasty: role of the posterior cruciate ligament. Clin Orthop Relat Res. 1996(331): 172-178, doi: 10.1097/00003086-199610000-00024, indexed in Pubmed: 8895635.

7. Chaudhary R, Beaupré LA, Johnston DWC. Knee range of motion during the first two years after use of posterior cruciate-stabilizing or posterior cruciate-retaining total knee prostheses. A randomized clinical trial. J Bone Joint Surg Am. 2008; 90(12): 2579-2586, doi: 10.2106/ JBJS.G.00995, indexed in Pubmed: 19047702.

8. Gigante A, Specchia N, Greco F. Age-related distribution of elastic fibers in the rabbit knee. Clin Orthop Relat Res. 1994(308): 33-42, doi:10.1097/00003086-19941100000006, indexed in Pubmed: 7955697.

9. Katonis PG, Assimakopoulos AP, Agapitos MV, et al. Mechanoreceptors in the posterior cruciate ligament. Histologic study on cadaver knees. Acta Orthop Scand. 1991; 62(3): 276-278, doi: 10.3109/17453679108993609, indexed in Pubmed: 2042472.

10. Kim YH, Choi Y, Kwon OR, et al. Functional outcome and range of motion of high-flexion posterior cruciateretaining and high-flexion posterior cruciate-substituting total knee prostheses. A prospective, randomized study. J Bone Joint Surg Am. 2009; 91(4): 753-760, doi: 10.2106/ JBJS.H.00805, indexed in Pubmed: 19339558.

11. Kleinbart FA, Bryk E, Evangelista J, et al. Histologic comparison of posterior cruciate ligaments from arthritic and age-matched knee specimens. J Arthroplasty. 1996; 11(6): 726-731, doi: 10.1016/s0883-5403(96)80012-x, indexed in Pubmed: 8884449. 
12. Lattanzio PJ, Chess DG, MacDermid JC. Effect of the posterior cruciate ligament in knee-joint proprioception in total knee arthroplasty. J Arthroplasty. 1998; 13(5): 580-585, doi: 10.1016/s0883-5403(98)90059-6, indexed in Pubmed: 9726325.

13. Nelissen RG, Hogendoorn PC. Retain or sacrifice the posterior cruciate ligament in total knee arthroplasty? A histopathological study of the cruciate ligament in osteoarthritic and rheumatoid disease. J Clin Pathol. 2001; 54(5): 381-384, doi: 10.1136/jcp.54.5.381, indexed in Pubmed: 11328838.

14. Pagnano MW, Cushner FD, Scott WN. Role of the posterior cruciate ligament in total knee arthroplasty. J Am Acad Orthop Surg. 1998; 6(3): 176-187, doi: 10.5435/00124635199805000-00006, indexed in Pubmed: 9682080.

15. Schultz RA, Miller DC, Kerr CS, et al. Mechanoreceptors in human cruciate ligaments. A histological study. J Bone Joint Surg Am. 1984; 66(7): 1072-1076, doi: 10.2106/00004623-198466070-00014, indexed in Pubmed: 6207177.

16. Schwab JH, Haidukewych GJ, Hanssen AD, et al. Flexion instability without dislocation after posterior stabilized total knees. Clin Orthop Relat Res. 2005; 440: 96-100, doi: 10.1097/00003086-200511000-00018, indexed in Pubmed: 16239790.

17. Shannon FJ, Cronin JJ, Cleary MS, et al. The posterior cruciate ligament-preserving total knee replacement: do we ,preserve' it? A radiological study. J Bone Joint Surg Br. 2007; 89(6): 766-771, doi: 10.1302/0301-620X.89B6. 18782, indexed in Pubmed: 17613501.

18. Simmons S, Lephart S, Rubash $\mathrm{H}$, et al. Proprioception following total knee arthroplasty with and without the posterior cruciate ligament. J Arthroplasty. 1996; 11(7):
763-768, doi: 10.1016/s0883-5403(96)80174-4, indexed in Pubmed: 8934314.

19. Skinner HB, Barrack RL. Joint position sense in the normal and pathologic knee joint. J Electromyogr Kinesiol. 1991; 1(3): 180-190, doi: 10.1016/1050-6411(91)90033-2, indexed in Pubmed: 20870508.

20. Straw R, Kulkarni S, Attfield S, et al. Posterior cruciate ligament at total knee replacement. Essential, beneficial or a hindrance? J Bone Joint Surg Br. 2003; 85(5): 671-674, doi: 10.1016/0968-0160(95)00022-4, indexed in Pubmed: 12892188.

21. Stubbs G, Dahlstrom J, Papantoniou P, et al. Correlation between macroscopic changes of arthrosis and the posterior cruciate ligament histology in the osteoarthritic knee. ANZ J Surg. 2005; 75(12): 1036-1040, doi: 10.1111/j.1445-2197.2005.03610.x, indexed in Pubmed: 16398804.

22. Swanik CB, Lephart SM, Rubash HE. Proprioception, kinesthesia, and balance after total knee arthroplasty with cruciate-retaining and posterior stabilized prostheses. J Bone Joint Surg Am. 2004; 86-A(2): 328-334, doi: 10.2106/00004623-200402000-00016, indexed in Pubmed: 14960678.

23. Udomkiat $P$, Meng BJ, Dorr LD, et al. Functional comparison of posterior cruciate retention and substitution knee replacement. Clin Orthop Relat Res. 2000(378): 192-201, doi: 10.1097/00003086-200009000-00029, indexed in Pubmed: 10986994.

24. Wang CJ, Wang JW, Chen HS. Comparing cruciate-retaining total knee arthroplasty and cruciate-substituting total knee arthroplasty: a prospective clinical study. Chang Gung Med J. 2004; 27(8): 578-585, indexed in Pubmed: 15553604. 